\title{
Observation of Inter-Bandgap States in Doped Ceria via Monochromated EELS
}

\author{
W.J. Bowman ${ }^{1}$, K. March $^{2}$, T. Aoki ${ }^{3}$, C.A. Hernandez ${ }^{1}$, P.A. Crozier ${ }^{1}$
}

1. School for the Engineering of Matter, Transport and Energy, Arizona State University, 501 E. Tyler Mall, Tempe Arizona 85287-6106

2. Laboratoire de Physique des Solides, Bâtiment 510, Université Paris-Sud, 91405 Orsay Cedex, France

3. LeRoy Eyring Center for Solid State Science, Arizona State University, Tempe, Arizona 85287-1704, USA

Recent advances in STEM EELS monochromation now allow for routine ultra-high energy resolution of $15 \mathrm{meV}$ or better in the low-loss region [1]. The ability to now correlate atomic structure and electronic structure in the low-loss and bandgap regions of the energy-loss spectrum represents a powerful tool for characterization of electronic and optical properties of material defects and device features such as grain boundaries and interfaces. Here, we demonstrate an application of monochromated low-loss EELS for characterizing the electronic transition from valence band to inter-bandgap states in $\operatorname{Pr}_{0.1} \mathrm{Ce}_{0.9} \mathrm{O}_{2-\delta}(\mathrm{PCO})$ nanoparticles, an ionic/electronic-conducting solid whose cations have readily accessible trivalent/tetravalent redox couples (i.e. $\mathrm{Ce}^{3+/ 4+}$ and $\operatorname{Pr}^{3+/ 4+}$ ).

The specimen for this study was heat-treated PCO nanoparticles $(\sim 30 \mathrm{~nm})$ synthesized in the authors' lab via spray drying (rapid solution evaporation), which resulted in partially oxygendeficient particles (fig. A). Subsequent heating for $3 \mathrm{hr}$ at $900{ }^{\circ} \mathrm{C}$ in air maximized the number of tetravalent cations (by filling $\mathrm{O}$ vacancies), causing the powder to appear dark red in color (fig. $\mathrm{B}$; see discussion below). Energy-loss spectra were acquired using a monochromated Nion UltraSTEM100 at $60 \mathrm{keV}$ in the energy-loss range up to $\sim 5 \mathrm{eV}$ using dispersions including $3 \mathrm{meV}$ and $5 \mathrm{meV}$. Under these conditions the zero-loss peak (ZLP) full width half maximum was typically $18 \mathrm{meV}$ to $25 \mathrm{meV}$ to provide reasonable signal-to-noise. Data were acquired using the standard transmission geometry as well as an aloof-beam configuration, whereby the STEM probe was positioned in the vacuum some distance from the specimen (fig. C) to avoid the radiation damage. Core-loss EELS and optical observations corroborated findings based on lowloss data.

An inverse power law fit was used to subtract the ZLP, and preliminary data (e.g. fig. D) revealed that the conduction band onset energy, i.e. the band gap, was approximately $3.2 \mathrm{eV}-$ measured from the intercept of the linear extrapolation of the conduction band edge with the energy-loss axis. Spectra showed a feature within the bandgap with a rising onset and extended plateau. The rise spanned the energy range of $1.6 \mathrm{eV}$ to $2.4 \mathrm{eV}$ and the feature's plateau extended from $2.4 \mathrm{eV}$ to the conduction band edge. In the core-loss region, the separation between the Ce and $\operatorname{Pr} \mathrm{M}_{5}$ white lines was monitored to identify the cations' oxidation state. Separations ranged from $46.2 \mathrm{eV}$ to $46.9 \mathrm{eV}$, and were typically greater than the separations measured in reference material containing $\mathrm{Ce}^{4+}$ and $\operatorname{Pr}^{3+}$ which were $46.3 \mathrm{eV}$ (see fig. E), which verified that the sample contained a substantial fraction of $\operatorname{Pr}^{4+}[2]$.

The inter-bandgap feature was attributed to transitions of electrons from the $\mathrm{O}-2 \mathrm{p}$ valence band into unoccupied Pr-4f levels lying slightly below the Ce-4f conduction band as shown in fig B. The energy range of the spectral feature's rising onset was assumed to be proportional to the 
Pr-4f band width, and the midpoint energy of the sloping onset $(2.0 \mathrm{eV})$ was taken to be the Pr-4f band center position. This inter-band state associated with Pr doping results in broad optical absorption above $\sim 2 \mathrm{eV}$, and causes the oxide to appear deep red in color [3]. This position and the measured energy of the $\mathrm{Ce}-4 \mathrm{f}$ conduction band onset (i.e. the direct optical bandgap) agreed closely with reports of optical bandgaps of PCO (2.1 eV) [3] and $\mathrm{CeO}_{2}$ (3.6 eV) [4]. Interestingly, the inter-band state persisted throughout time-resolved acquisitions, likely indicating signal delocalization in transmission mode.

\section{References and acknowledgements}

1. O.L. Krivanek et al. Nature 514, 209-212 (2014).

2. C. López-Cartes et al. Chem. Commun. 5, 644-645 (2003).

3. J.J. Kim et al. Chem. of Mater. 26, 1374-1379 (2014).

4. E. Ruiz-Trejo. Phys. Chem. Solids. 74, 605-610 (2013).

5. W.J.B. would like to acknowledge the NSF's Graduate Research Fellowship (DGE-1211230) for continued financial support. We gratefully acknowledge support of NSF grant DMR-1308085 and ASU's John M. Cowley Center for High Resolution Electron Microscopy.

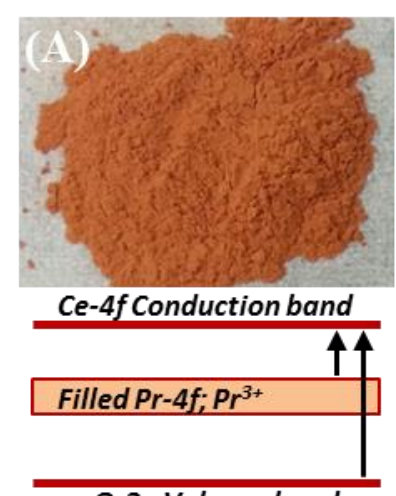

$0-2 p$ Valence band
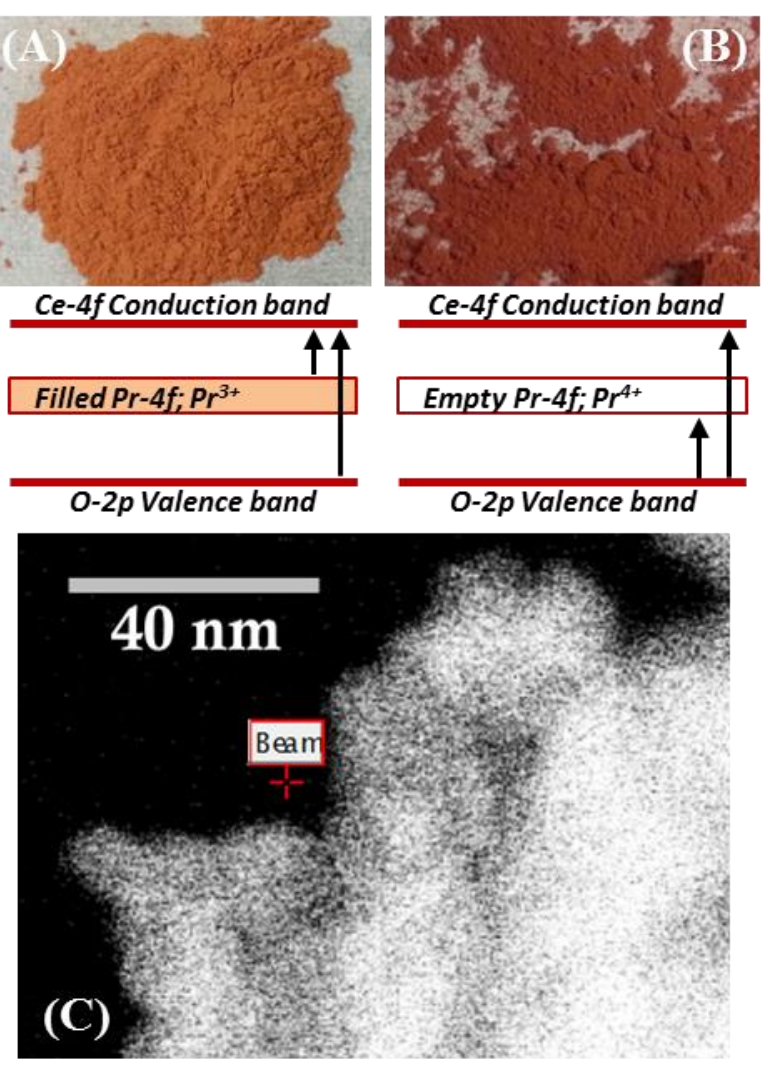

$0-2 p$ Valence band

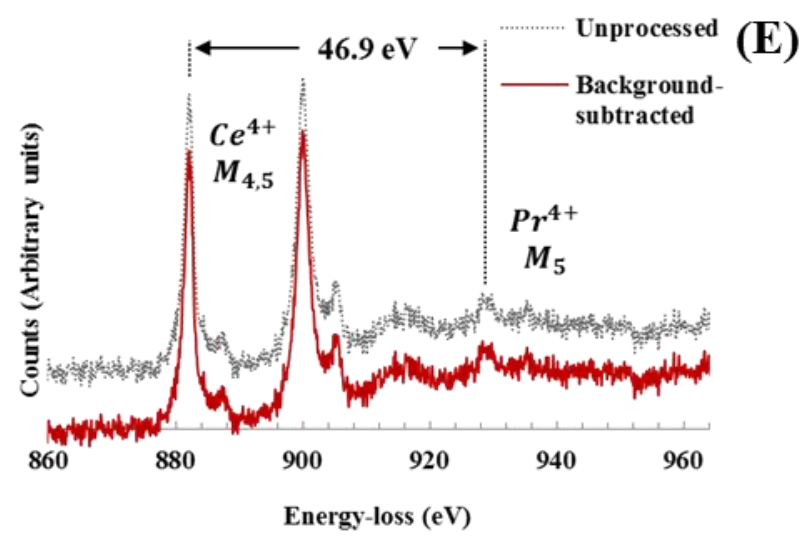

Figs. A \& B. PCO (A) as-synthesized and (B) after heating. Band diagrams adapted from [2]. Fig. C. Representative HAADF STEM image illustrating aloof-beam EELS configuration.

Fig. D. Low-loss transmission EELS of Pr-4f inter-band state, and ceria conduction band onset. Fig. E. Core-loss EELS of Ce and $\operatorname{Pr~M}_{4,5}$ white lines in $\mathrm{Ce}^{4+} / \mathrm{Pr}^{4+}$ condition. 